\title{
SCHWANNOMAS OF THE GASTROINTESTINAL TRACT
}

\author{
Beatrice Lintoiu' ${ }^{1}$ Irina Balescu ${ }^{2}$, Nicolae Bacalbasa ${ }^{3}$ \\ 1 "Profesor Dr. Agripa Ionescu" Clinical Emergency Hospital, Bucharest, Romania \\ 2 "Ponderas"Hospital, Bucharest, Romania \\ 3 "Carol Davila" University of Medicine and Pharmacy, Bucharest, Romania
}

\begin{abstract}
Schwannomas are rare tumors originating from the Schwann cells, that form the neural sheath. These tumors occur most frequently in the head, neck, arms and limbs. Primary schwannomas of the colon and rectum are extremely rare. They are usually benign. Pre-operative biopsy examinations may be difficult and immunohistochemistry is necessary for the correct diagnosis. In contrast to gastrointestinal stromal tumors, schwannomas are negative for CD117 and positive for S100 protein and vimentin. In extremely rare cases, they can present with malignant degeneration if not surgically removed. Therefore, the gold standard treatment for schwannomas is surgical resection.
\end{abstract}

Keywords: schwannoma, immunohistochemistry, colon, surgery, S-100 protein

\section{INTRODUCTION}

There are many synonyms for neurogenic tumors of the gastrointestinal tract, such as schwannoma, neurinoma, neurogenic fibroma, neurofibroma, neurilemoma and plexiform neurofibromatosis. Usually, this different names are used to relate them to the structure from which they originate. The correct name of gastrointestinal schwannoma is based on the fact that this tumors originate from Schwann cells in the neurons of the myenteric plexus $(1,2,3)$.

Schwannomas were first described by Verocay in 1910. They represent a group of benign tumours derived from the Schwann cells which form the neural sheath. Commonly they occurs in the peripheral nerve of the limbs or body, spinal cord, and central nervous system Aside from cranial nerves I and II, which do not include Schwann cells, schwannomas can arise from any nerve sheath, the most common type of benign schwannoma being the acoustic neuroma (VIII cranial nerve) $(4,5)$. The incidence is similar for males and females and even if they can develop at any age, the sixth and seventh decades of life are the most commonly involved. There has been an increase in reports of mesenchymal tumors in recent years with the advent of newer immunohistochemical staining, but primary schwannomas located in the colon and rectum are extremely rare (2-6\% of all mesenchymal tumors of digestive tract) when they are not associated with inherited syndrome such as von Recklinghausen's (or type 1) neurofibromatosis (NF-1), multiple endocrine neoplasia type 2B (MEN 2B) or Cowden syndrome. There are approximately 50 published cases reported in the literature worldwide $(3,6,7,8)$.

Stomach and small intestine are the most common digestive sites for schwannomas (83 and 12\%, respectively). In the large intestine, cecum is the most common location, followed by sigmoid colon, transverse colon, descending colon, and rectum $(6,8,9)$. In contrast, in a review of the Japanese literature, including 46 cases, Inagawa et al. (10) reported rectum $(45.7 \%)$ to be the most frequent site of occurrence. They also calculated the incidence of schwannoma arising from the right colon, excluding the appendix, at $19.6 \%$ (9 cases among forty-six) of cases of colon and rectal schwannomas.

\section{DIAGNOSIS}

Schwannomas are mostly asymptomatic. Depending on tumor size and location, schwannomas of the colon may occasionally produce symptoms, such as constipation, bleeding, abdominal pain or discomfort and anal pain (11).

Corresponding author:

Nicolae Bacalbasa, 2 Dimitrie Racovita Street, Bucharest, Romania

E-mail: nicolae_bacalbasa@yahoo.ro 
Colonoscopy, abdominal ultrasound, abdominal $\mathrm{CT}$ and abdominal magnetic resonance imaging (MRI) are important in evaluating the contours of colorectal schwannomas, their relationship with the surrounding organs, tumor multiplicity and metastasis. Levy et al. (12) described gastrointestinal schwannomas as homogeneously attenuated and well-defined mural masses on CT, noting that they were indistinguishable from gastrointestinal stromal tumors.

Colonic schwannomas have intense avidity for flourodeoxy D-glucose (FDG) similar to malignant gastrointestinal stromal tumors hence are indistinguishable on Positron emission tomography (13). In accessible sites endosonographic features of irregular extraluminal margins, cystic spaces, and lymph node with malignant features may help differentiate benign tumors from the malignant ones. Even if endoscopic ultrasonography (EUS) surpassed other imaging techniques for diagnosis purposes, it can not differentiate a schwannoma from other gastrointestinal stromal tumors. EUS-guided fine needle aspiration or biopsy of submucosal tumors has been attempted to increase the diagnosis accuracy (14). A review by Tedeschi et al. (15) noted that the biopsy specimens for the diagnosis of schwannomas are limited due to the submucosal growth of the tumour.

Since schwannomas are tumors growing from the neural sheath, in the digestive tract they arise from the autonomous nervous system; more frequently from Auerbach's plexus and less frequently from Meissner's plexus. Neoplasms originating from Auerbach's plexus protrude into the intestinal lumen and are characterized by a non-pedunculated oval-shaped mass, whereas those arising from Meissner's plexus are often pedunculated polyps $(11,16)$.

Schwannomas tend to share the gross morphologic features with other submucosal tumors and may be hard, solid, ulcerated, or calcified in rare cases (17). Histologically, schwannomas are characterized by Verocay corpuscles, a lymphoid cuff and a spiral-like form consisting of densely arrayed spindle-shaped cells, palisade arrangements and loose reticular networks of cells. Two histological growth patterns have been described: Antoni A (dense growth of fusiform cells compactly arranged in palisades to form Verocay bodies) and Antoni B (the fusiform cells are more loosely distributed with rounded or elongated nuclei, with a great quantity of myxoid stroma and xanthomatous histiocytes). Schwann cells covering a basal membrane are not generally identified $(8,18)$.
Although certain studies consider schwannomas to be a subtype of gastrointestinal stromal tumor (GIST) belonging to the gastrointestinal autonomic nerve tumors (GANT), there are significant histopathological and immunohistochemical differences $(4,11)$. In contrast to GISTs, schwannomas are consistently negative for CD117 (KIT), usually negative for CD34, CKs, smooth muscle actin and desmin, but strongly positive for S100 protein and vimentin $(6,7,16)$. Lymphoid cuff, diffuse lymphoid infiltration, focal nuclear atypia, a microtrabecular pattern and an impression of cellular heterogeneity are not features of GISTs. Smooth muscle tumors typically have desmin and smooth muscle actin reactivity, whereas gastrointestinal stromal tumor shows immunopositivity to CD34 and CD117 but not to S-100 protein (8). A combinatorial assay of S-100, CD34 and CD117 proteins, therefore, is definitive in differentiating schwannoma from GISTs.

Inagawa et al. (10) demonstrated that the rate of accurate preoperative diagnosis for benign schwannomas was only $15.2 \%$ (seven of forty-six cases) and that the most common preoperative diagnosis was submucosal tumor $(39.1 \%)$. The reasons for inaccurate preoperative diagnosis can include a limited knowledge of such tumors and insufficient volume of biopsy specimen for pathologic confirmation. In the absence of adequate preoperative diagnosis, the extent of excision should be determined by the characteristics of the tumor such as size, location, and consistency, as was suggested by Braumann et al (7).

Even if primary schwannomas are in their majority benign tumors, the possibility of malignant degeneration exists and is directly related to the tumor dimensions. Radical excision with margins free of disease is the treatment of choice, since the response to chemotherapy and radiotherapy remains uncertain. Despite aggressive surgical management, these tumors may appear with a high rate of local recurrence. In such cases, there are limited treatment options and the prognosis is poor $(4,18)$.

Das Gupta and Brasfield (3) reported that in 2\% of cases, distant metastasis may be observed. A correct histological diagnosis requires investigation of the Ki-67 proliferative index (MIB-1), as its positivity $(\geq 5 \%)$ is correlated with greater tumor aggressiveness. A mitotic activity rate of more than five mitoses per field at high magnification along with tumor size $>5 \mathrm{~cm}$ are associated with a high risk of metastasis and recurrence. Benign lesions are characterized by a low rate of mitosis and the absence of atypical mitotic figures and nuclear hyperpigmentation $(6,18)$. 
Hemorrhage, necrosis and ulceration are more frequently characteristics of malignant schwannomas and are often used to macroscopically differentiate malignancy from benignity. Even if many parameters have been studied in relation to tumor behavior, so far no single parameter alone allows sufficient prediction of the degree of malignity.

Currently, the gold standard treatment for schwannomas is complete surgical resection. In the case of the malignant schwannoma, oncological resection is indicated. Some authors suggests that, even if the lymph nodes are not involved, radical surgery is the best treatment. Others considers that simple enucleation may be sufficient due to its benign nature. Minimally invasive surgery using videolaparoscopic access may be indicated for tumors located in the colon and rectum. For tumors in the distal rectum, transanal endoscopic resection can be a feasible therapeutic option (18-21). Even if radiotherapy has been shown to decrease tumor growth and regression in neurogenic schwannoma, the response to chemotherapy and radiotherapy remains uncertain in gastrointestinal schwannomas. As for the survival, the cases of malignant schwannoma that have been reported in the literature have

\section{REFERENCES}

1. Mir R., Singh V.P., Kaul S. Varied presentation of schwannoma - a case study. Case Rep Oncol 2010; 3:354-61

2. Hou Y.Y., Tan Y.S., Xu J.F., et al. Schwannoma of the gastrointestinal tract: a clinicopathological, immunohistochemical and ultrastructural study of 33 cases. Histopathology 2006; 48:536-45

3. Das Gupta T.K., Brasfield R.D. Tumors of peripheral nerve origin: benign and malignant solitary schwannomas. CA Cancer J Clin.1970; 20:228-233

4. Fotiadis C.I., Kouerinis I.A., Papandreou I., Zografos G.C., Agapitos G. Sigmoid schwannoma: a rare case. World J Gastroenterol. 2005; 11:5079-5081

5. Sanmillan J.L., Plans G., Vidal N., Acebes J.J. Supratentorial brain schwannomas: an uncommon location for a common tumour. Br J Neurosurg. 2014; 28:25-28

6. Nonose R., Lahan A.Y., Santos Valenciano J., Martinez C.A. Schwannoma of the colon. Case Rep Gastroenterol. 2009; 3:293-299

7. Braumann C., Guenther N., Menenakos C., Junghans T. Schwannoma of the colon mimicking carcinoma: a case report and literature review. Int J Colorectal Dis. 2007; 22:1547-1548

8. Miettinen M., Shekitka K.M., Sobin L.H. Schwannomas in the colon and rectum: a clinicopathologic and immunohistochemical study of 20 cases. Am J Surg Pathol. 2001; 25:846-55

9. Park K.J., Kim K.H., Roh Y.H., Kim S.H., Lee J.H., Rha S.H., et al. Isolated primary schwannoma arising on the colon: report of two cases and review of the literature. J Korean Surg Soc. 2011; 80:367-372

10. Inagawa S., Hori M., Shimazaki J., et al. Solitary schwannoma of the colon: report of two Cases. Surgery Today. 2001; 31(9):833-838

11. Zippi M., Pica R., Scialpi R., Cassieri C., Avallone E.V., Occhigrossi G. Schwannoma of the rectum: A case report and literature review. World J Clin Cases. 2013; 1:49-51

12. Levy A.D., Quiles A.M., Miettinen M., Sobin L.H. Gastrointestinal schwannomas: CT features with clinicopathologic correlation. AJR Am J Roentgenol. 2005; 184:797-802 a poor prognosis accompanied by a rapid clinical course and a poor response to chemotherapy, with an average life span of two years.

\section{CONCLUSIONS}

Schwannoma represents a pathological entity that can mimic colonic adenocarcinoma and may cause diagnostic and treatment dilemmas. The best therapeutic option is complete surgical removal with margins free from tumor involvement. Because the risk of malignant transformation is small, broad lymph node resections are not recommended. The surgical approach depends on the size and location of the tumor and on its histopathologic pattern. The use of radiotherapy or adjuvant chemotherapy has conflicting results and is not recommended for routine use. There is insufficient evidence to determine the total period of follow-up and whether endoscopic surveillance improves the patient outcome.

Although rare, schwannomas must be included in the differential diagnosis from other intestinal mesenchymal neoplasms, such as smooth muscle tumors, neurofibromas, and GISTs.

13. Wang C.L., Neville A.M., Wong T.Z., Hall A.H., Paulson E.K., Bentley R.C. Colonic schwannoma visualized on FDG PET/CT. Clinical Nuclear Medicine. 2010; 35(3):181-183

14. Larghi A., Verna E.C., Ricci R., Seerden T.C., Galasso D., Carnuccio A., Uchida N., Rindi G., Costamagna G. EUS-guided fine-needle tissue acquisition by using a 19-gauge needle in a selected patient population: a prospective study. Gastrointest Endosc. $2011 ; 74: 504-510$

15. Tedeschi M., Cuccia F., Angarano E., et al. Solitary schwannoma of the rectum mimicking rectal cancer. Ann Ital Chir 2011; 82:309-12

16. Tsunoda C., Kato H., Sakamoto T., Yamada R., Mitsumaru A., et al. A case of benign schwannoma of the transverse colon with granulation tissue. Case Rep Gastroenterol. 2009; 3:116-120

17. Tomozawa S., Masaki T., Matsuda K., Yokoyama T., Ishida T., Muto T. A schwannoma of the cecum: case report and review of japanese schwannomas in the large intestine. J Gastroenterol. 1998; 33(6):872-875

18. Bugiantella W., Rondelli F., Mariani L., Peppoloni L. , Cristallini E., Mariani E. Schwannoma of the colon: A case report. Oncol Lett. 2014 Dec; 8(6):2511-2512

19. Baskaran V., Cho W.S. , Perry M. Rectal schwannoma on endoscopic polypectomy. BMJ Case Rep. 2014

20. Kanneganti K., Patel H., Niazi M., Kumbum K., Balar B. Cecal Schwannoma: A Rare Cause of Gastrointestinal Bleeding in a Young Woman with Review of Literature. Gastroenterol Res Pract. 2011; 2011: 142781

21. Agaimy A., Märkl B., Kitz J., et al. Peripheral nerve sheath tumors of the gastrointestinal tract: a multicenter study of 58 patients including NF1-associated gastric schwannoma and unusual morphologic variants. Virchows Arch. 2010; 456:411-422 